\title{
The Emergence of Spacetime from the Quantum in Three Steps
}

\author{
Mohamed S. El Naschie \\ Department of Physics, Faculty of Science, University of Alexandria, Alexandria, Egypt \\ Email: Chaossf@aol.com
}

Received 23 May 2016; accepted 28 May 2016; published 31 May 2016

Copyright (C) 2016 by author and Scientific Research Publishing Inc.

This work is licensed under the Creative Commons Attribution International License (CC BY). http://creativecommons.org/licenses/by/4.0/

(c) (;) Open Access

\begin{abstract}
The paper presents a very simple and straight forward yet pure mathematical derivation of the structure of actual spacetime from quantum set theory. This is achieved by utilizing elements of the topological theory of cobordism and the Menger-Urysohn dimensional theory in conjunction with von Neumann-Connes dimensional function of Klein-Penrose modular holographic boundary of the E8E8 exceptional Lie group bulk of our universe. The final result is a lucid sharp mental picture, namely that the quantum wave is an empty set representing the surface, i.e. boundary of the zero set quantum particle and in turn quantum spacetime is simply the boundary or the surface of the quantum wave empty set. The essential difference of the quantum wave and quantum spacetime is that the wave is a simple empty set while spacetime is a multi-fractal type of infinitely many empty sets with increasing degrees of emptiness.
\end{abstract}

\section{Keywords}

Quantum Spacetime, Transfiite Theory, Noncommutative Geometry, 'tHooft-Susskind Holography, Cantorian Spacetime, Penrose-Connes Fractal Universe, E-Infinity Theory, E8 Exceptional Lie Group

\section{Introduction}

Renown Austrian writer Ferdinand Kürnberger [1] once wrote “...and whatever a man knows, whatever is not mere rambling and roaring that he has heard, can be said in three words”. It seems that these words made such a strong impression on his fellow compatriot and Cambridge Professor, Ludwig Wittgenstein [2] that he made it to the motto of his most famous book "Tractatus Logico-Philosophicus" [2].

In the present short paper the author goes even further than Wittgenstein by taking the three words to literally mean three steps leading to the emergence of spacetime from the quantum [3]-[24]. It is the main aim of the 
present analysis to give these three steps in an irreducibly simple way. To keep this simplicity as well as a short presentation we had to include a large selection of references [1]-[84]. In particular refs. [44]-[89] may be regarded as supplementary literature which may be skipped over at first reading and considered in depth only later at a second reading of the present paper.

\section{The Emergence of Spacetime from the Quantum}

The following derivation consists of exactly three steps as alluded to in our short introduction:

\section{Step 1}

We start with the quantum pre-particle as modelled by the zero set [3]. Consequently following the dimensional recursive function of von Neumann-Connes (see Appendix 1 for details), we have for the quantum particle [3] [5] [20]

$$
D(O) \equiv(0, \phi)
$$

where the zero is the Menger-Urysohn topological dimension and $\phi=2 /(1+\sqrt{5})$ is the Hausdorff dimension of the zero set.

\section{Step 2}

From step one it follows naturally that the surface of $\mathrm{D}(\mathrm{O})$ is given by the empty set [3]-[6] [36] [37]. Consequently the quantum wave as the cobordism of the quantum particle can be modelled by the empty set. This is given by the same dimensional function of von Neumann-Connes as [3] [20] [26]

$$
D(-1)=\left(-1, \phi^{2}\right) \text {. }
$$

A short explanation for the negative minus one topological dimension of the empty set is given in Appendix 2 .

\section{$\underline{\text { Step } 3}$}

Now it may come as a slight surprise that continuing in the same manner as above, the surface of the quantum wave turns out to be nothing else but our quantum spacetime [3] [25] [26]

$$
D(-2)=\left(-2, \phi^{3}\right) \text {. }
$$

In other words, quantum spacetime is an emptier set than the empty set. Not only that but the average empty set from $D(-1)$ to $D(-\infty)$ turns out to have on average a Hausdorff dimension equal $\phi^{3}$ [3] [25] [26]. This follows neatly from the fact that the expectation value of quantum spacetime is given by the Hausdorff dimension of Cantorian spacetime $4+\phi^{3}$ [43] [57]. Therefore the average empty set dimension must be the reciprocal value of $4+\phi^{3}$ which is $\phi^{3}$. This proves the correctness of what we stated above [3]-[6] [36] [37].

The preceding three steps give the quintessence of our theory and explain both the quantum wave and quantum spacetime in one stroke in terms of each other [3]. Quantum spacetime is simply the surface of the quantum wave in exactly the same manner as the quantum wave is the surface of the quantum particle. Thus quantum wave and quantum spacetime are basically more or less the very same substance or said more subtly, the very same "non-substance" [27] [42]. Implications for "tHooft-Susskind holographic theory and the black hole information paradox will not be discussed here [18] [19].

\section{The Hausdorff Dimension of Space, Time and Spacetime}

Let us consider the dimensions corresponding to the unfolding of our three basic steps or basic sets.

\section{Set 1}

Unfolding the zero set with which we mean moving from the negative topological dimensions domain to the positive one by inversion [3]-[10] one finds

$$
D=1 / \phi=1+\phi=1.618033989
$$

This we interpret as a one dimensional classical string plus an irrational tail $(\phi)$. In other words $D=1+\phi$ represents what we may call a fractal string.

\section{Set 2}

In analogy to the preceding zero set, our empty set leads to [3]-[10]

$$
D=1 / \phi^{2}=2+\phi=2.61833989
$$


This may be interpreted as a classical world sheet plus an irrational tail. Again this may be seen as a fractal world sheet [3]-[10]. It is remarkable how the intersection as well as the union of the fractal strings and the fractal world sheet span the E-infinity Cantorian space modelling quantum spacetime because [43] [57]

$$
(1 / \phi)+\left(1 / \phi^{2}\right)=4+\phi^{3}=1 / \phi^{3}
$$

as well as

$$
(1 / \phi) \otimes\left(1 / \phi^{2}\right)=4+\phi^{3}=1 / \phi^{3} .
$$

Consequently we conclude from the above that there is an intrinsic indistinguishability latent in our Cantorian manifold modelling quantum spacetime with regard to the operations of union and intersection, which explains the superficially paradoxical outcome of the two-slit experiment with quantum particles [71]. In fact we can reason that time is a fractal phenomena of our "space-time" manifold. The simplest way to show this is to consider the average zero-like set and empty set with Hausdorff dimension ranging from zero to $\phi$. This is easily found to be $\left\langle d_{c}^{(0)}\right\rangle=1 / 2$ which is a sort of coarse graining zero set [39]. Inserting into the expectation formula for the topological dimension and the Hausdorff dimension we find for the topological case [39] [40]

$$
\langle n\rangle=\frac{1+\left\langle d_{c}^{(0)}\right\rangle}{1-\left\langle d_{c}^{(0)}\right\rangle}=\frac{1+(1 / 2)}{1-(1 / 2)}=3
$$

and for the Hausdorff counterpart

$$
\left\langle d_{c}\right\rangle=\frac{1}{\left\langle d_{c}^{(0)}\right\rangle\left(1-\left\langle d_{c}^{(0)}\right\rangle\right)}=\frac{1}{(1 / 2)(1-(1 / 2))}=4
$$

respectively. The time dimension is consequently the difference between the two:

$$
D(\text { time })=\left\langle d_{c}\right\rangle-\langle n\rangle=4-3=1
$$

The preceding remarkable result could be used to elucidate the strong link between number theory and physics. We could for instance argue that our classical 3D space is simply an "integer" approximation of the basic two dimensions

$$
1+\phi \simeq 1
$$

and

$$
2+\phi \simeq 2
$$

leading to

$$
D(\text { space })=1+2 \simeq 3
$$

On the other hand Einstein's 4D could be seen as a rational approximation

$$
1+\phi \simeq 1.5
$$

and

$$
2+\phi \simeq 2.5
$$

leading to

$$
D(\text { spacetime })=1.5+2.5=4
$$

An even more striking feature of the deep relation between number theory as well as transfinite set theory and physics as seen through the mathematics of our present analysis is the following result which follows from the inversion of the zero set and empty set at the averaging level [39]

$$
\left\langle d_{c}^{(0)}\right\rangle=\phi \rightarrow\left\langle d_{c}^{(0)}\right\rangle=1 / 2
$$

and 


$$
\left\langle d_{c}^{(-1)}\right\rangle=\phi^{2} \rightarrow\left\langle d_{c}^{(-1)}\right\rangle=1 / 4
$$

This leads clearly to

$$
1 /\left\langle d_{c}^{(0)}\right\rangle=2
$$

and

$$
1 /\left\langle d_{c}^{(-1)}\right\rangle=4
$$

In other words the empty set quantum wave gives us directly the topological dimension of spacetime while the zero set particle gives us the topological dimension of the spacetime world sheet [3]-[10] [43] [48]. Clearly two world sheets corresponding to two quantum particles will give rise to spacetime dimensions when interacting whether by union, i.e. addition rule or intersection, i.e. multiplication rule because of the unique although trivial equation

$$
D=2+2=(2)(2)=4
$$

There are no other two integers which could stimulate the basic interaction of our two irrational numbers $1+\phi$ and $2+\phi$ except 2 and 2 as we pointed out on earlier occasions in more detail [38] [43]. However we feel that the present discussion which started with von Neumann-Connes recursive Fibonacci-like dimensional function (see Appendix 1) is mathematically much deeper and testifies for what we call post modernistic physics with which we anticipate a new era in physics were pure mathematics and real physics are one and the same thing [3].

\section{Conclusion}

“God made the bulk 'but' the surface was invented by the devil”. This is a well known quotation ascribed to Wolfgang Pauli which may be viewed as the theme for the present work. On the other hand the present work showed how the word bulk could be replaced by the quantum particle and then concluded that the quantum wave is simply the "surface" of this particle. Going one step further it was shown here in unheard of simplicity that spacetime is the multilayer (multi-fractal) surface of the quantum wave. This demonstrates how in three simple steps spacetime emerges from the quantum. Seen that way the surface as well as 'tHooft-Susskind holography is definitely not an invention by the devil but a great idea of deep, subtle beauty worthy of the great pure mathematician who created existence.

\section{References}

[1] Ferdinand Kürnberger: Encyclopaedia Britannica. http://www.britannica.com/biography/Ferdinand-Kurnberger

[2] Wittgenstein, L. (1961) Tractatus Logico-Philosphicus. Routledge and Kegan Paul, London.

[3] El Naschie, M.S. (2015) An Exact Mathematical Picture of Quantum Spacetime. Advances in Pure Mathematics, 5, 560-570. http://dx.doi.org/10.4236/apm.2015.59052

[4] El Naschie, M.S. (2015) If Quantum “Wave” of the Universe then Quantum "Particle” of the Universe: A Resolution of the Dark Energy Question and the Black Hole Information Paradox. International Journal of Astronomy \& Astrophysics, 5, 243-247. http://dx.doi.org/10.4236/ijaa.2015.54027

[5] El Naschie, M.S. (2015) On a Non-Perturbative Quantum Relativity Theory Leading to a Casimir-Dark Energy Nanotech Reactor Proposal. Open Journal of Applied Science, 5, 313-324. http://dx.doi.org/10.4236/ojapps.2015.57032

[6] El Naschie, M.S. (2015) From Fusion Algebra to Cold Fusion or from Pure Reason to Pragmatism. Open Journal of Philosophy, 5, 319-326. http://dx.doi.org/10.4236/ojpp.2015.56040

[7] El Naschie, M.S. (2013) Topological-Geometrical and Physical Interpretation of the Dark Energy of the Cosmos as a “Halo” Energy of the Schrodinger Quantum Wave. Journal of Modern Physics, 4, 591-596. http://dx.doi.org/10.4236/jmp.2013.45084

[8] El Naschie, M.S. (2016) From Witten's 462 Supercharges of 5-D Branes in Eleven Dimensions to the 95.5 Percent Cosmic Dark Energy Density behind the Accelerated Expansion of the Universe. Journal of Quantum Information Science, 6, 57-61. http://dx.doi.org/10.4236/jqis.2016.62007

[9] El Naschie, M.S. (2016) Negative Norms in Quantized Strings as Dark Energy Density of the Cosmos. World Journal 
of Condensed Matter Physics, 6, 63-67. http://dx.doi.org/10.4236/wjcmp.2016.62009

[10] El Naschie, M.S. (2016) On a Quantum Gravity Fractal Spacetime Equation: QRG HD + FG and Its Application to Dark Energy-Accelerated Cosmic Expansion. Journal of Modern Physics, 7, 729-736. http://dx.doi.org/10.4236/jmp.2016.78069

[11] El Naschie, M.S. (2016) Einstein-Rosen Bridge (ER), Einstein-Podolski-Rosen Experiment (EPR) and Zero Measure Rindler KAM Cantorian Spacetime Geometry (ZMG) Are Conceptually Equivalent. Journal of Quantum Information Science, 6, 1-9. http://dx.doi.org/10.4236/jqis.2016.61001

[12] El Naschie, M.S. (2016) Einstein’s Dark Energy via Similarity Equivalence, ‘tHooft Dimensional Regularization and Lie Symmetry Groups. International Journal of Astronomy \& Astrophysics, 6, 56-81. http://dx.doi.org/10.4236/ijaa.2016.61005

[13] El Naschie, M.S. (2016) Quantum Dark Energy from the Hyperbolic Transfinite Cantorian Geometry of the Cosmos. Natural Science, 8, 152-159. http://dx.doi.org/10.4236/ns.2016.83018

[14] El Naschie, M.S. (2015) Hubble Scale Dark Energy Meets Nano Scale Casimir Energy and the Rational of Their T-Duality and Mirror Symmetry Equivalence. World Journal of Nano Science and Engineering, 5, 57-67. http://dx.doi.org/10.4236/wjnse.2015.53008

[15] El Naschie, M.S. (2014) Cosmic Dark Energy from 'thooft's Dimesnionl Regularization and Witten’s Topological Quantum Field Pure Gravity. Journal of Quantum Information Science, 4, 83-91. http://dx.doi.org/10.4236/jqis.2014.42008

[16] El Naschie, M.S. (2015) Application of Dvoretzky’s Theorem of Measure Concentration in Physics and Cosmology. Open Journal of Microphysics, 5, 11-15. http://dx.doi.org/10.4236/ojm.2015.52002

[17] El Naschie, M.S. (2015) A Resolution of the Black Hole Information Paradox via Transfinite Set Theory. World Journal of Condensed Matter Physics, 5, 249-260. http://dx.doi.org/10.4236/wjcmp.2015.54026

[18] El Naschie, M.S. (2015) The Counterintuitive Increase of Information due to Extra Spacetime Dimensions of a Black Hole and Dvoretzky's Theorem. Natural Science, 7, 483-487. http://dx.doi.org/10.4236/ns.2015.710049

[19] El Naschie, M.S. (2014) Entanglement of E8E8 Exceptional Lie Symmetry Group Dark Energy, Einstein’s Maximal Total Energy and the Hartle-Hawking No Boundary Proposal as the Explanation for Dark Energy. World Journal of Condensed Matter Physics, 4, 74-77. http://dx.doi.org/10.4236/wjcmp.2014.42011

[20] Marek-Crnjac, L. (2015) On El Naschie’s Fractal-Cantorian Space-Time and Dark Energy-A Tutorial Review. Natural Science, 7, 581-598. http://dx.doi.org/10.4236/ns.2015.713058

[21] El Naschie, M.S. (2013) The Quantum Gravity Immirzi Parameter-A General Physical and Topological Interpretation. Gravitation and Cosmology, 19, 151-155. http://dx.doi.org/10.1134/S0202289313030031

[22] El Naschie, M.S. (2016) Cosserat-Cartan and de Sitter-Witten Spacetime Setting for Dark Energy. Quantum Matter, 5, 1-4. http://dx.doi.org/10.1166/qm.2016.1247

[23] El Naschie, M.S. (2015) The Self Referential Pointless Universe Geometry as the Key to the Resolution of the Black Hole Information Paradox. International Journal of Innovation in Science and Mathematics, 3, 254-265.

[24] El Naschie, M.S. (2016) On a Fractal Version of Witten’s M-Theory. Journal of Astronomy \& Astrophysics, 6, 135144. http://dx.doi.org/10.4236/ijaa.2016.62011

[25] Connes, A. (1994) Noncommutative Geometry. Academic Press, San Diego, See in Particular 85-93.

[26] Marek-Crnjac, L. (2011) The Hausdorff Dimension of the Penrose Universe. Physics Research International, 2011, Article ID: 874302. http://dx.doi.org/10.1155/2011/874302

[27] Connes, A., Lichnerowicz, A. and Schützenberger, M.P. (2001) Triangle of Thought. American Mathematical Society, Providence.

[28] Changeux, J.P. and Connes, A. (1995) Conversations on Mind, Matter and Mathematics. Princeton University Press, Princeton.

[29] Marcolli, M. (2010) Feynman Motives. World Scientific, Singapore.

[30] Connes, A. and Marcolli, M. (2008) Quantum Fields and Motives. American Mathematical Society, USA.

[31] Scheck, F., Upmeier, H. and Werner, W. (Editors) (2002) Noncommutative Geometry and the Standard Model of Elementary Particle Physics. Springer, Berlin. http://dx.doi.org/10.1007/3-540-46082-9

[32] Landi, G. (1997) An Introduction to Noncommutative Spaces and Their Geometrics. Springer, Berlin, See in Particular 73-77.

[33] El Naschie, M.S. (1998) Penrose Universe and Cantorian Spacetime as a Model for Noncommutative Quantum Geometry. Chaos, Solitons \& Fractals, 9, 931-933. http://dx.doi.org/10.1016/S0960-0779(98)00077-0 
[34] El Naschie, M.S. (1998) Von Neumann Geometry and E-Infinity Quantum Spacetime. Chaos, Solitons \& Fractals, 9, 2023-2030.

[35] Goldfain, E. (2004) On a Possible Evidence for Cantorian Space-Time in Cosmic Ray Astrophysics. Chaos, Solitons \& Fractals, 20, 427-435. http://dx.doi.org/10.1016/j.chaos.2003.10.012

[36] He, J.-H., Zhong, T., Xu, L., Marek-Crnjac, L., Nada, S.I. and Helal, M.A. (2011) The Importance of the Empty Set and Noncommutative Geometry in Underpinning the Foundations of Quantum Physics. Nonlinear Science Letters B, 1, 14-23.

[37] Marek-Crnjac, L. (2011) The Physics of Empty Sets and the Quantum. Nonlinear Science Letters B, 1, 4-5.

[38] Mandelbrot, B. (1990) Negative Fractal Dimensions and Multifractals. Physica A: Statistical Mechanics and Its Applications, 163, 306-315. http://dx.doi.org/10.1016/0378-4371(90)90339-T

[39] El Naschie, M.S. (1996) On Numbers, Probability and Dimensions. Chaos, Solitons \& Fractals, 7, 955-959. http://dx.doi.org/10.1016/0960-0779(96)00036-7

[40] El Naschie, M.S. (1994) On Certain "Empty” Cantor Sets and Their Dimensions. Chaos, Solitons \& Fractals, 4, 293296. http://dx.doi.org/10.1016/0960-0779(94)90152-X

[41] El Naschie, M.S. (1993) Statistical Mechanics of Multi-Dimensional Cantor Sets, Gödel Theorem and Quantum Spacetime. Journal of the Franklin Institute, 330, 199-211. http://dx.doi.org/10.1016/0016-0032(93)90030-X

[42] El Naschie, M.S. (2006) New Hot Paper Comments. ESI Special Topics, Thomson Essential Science Indicators, September.

[43] El Naschie, M.S. (2004) A Review of E-Infinity Theory and the Mass Spectrum of High Energy Particle Physics. Chaos, Solitons \& Fractals, 19, 209-236. http://dx.doi.org/10.1016/S0960-0779(03)00278-9

[44] El Naschie, M.S. (1998) On the Uncertainty of Cantorian Geometry and the Two-Slit Experiment. Chaos, Solitons \& Fractals, 19, 517-529. http://dx.doi.org/10.1016/S0960-0779(97)00150-1

[45] El Naschie, M.S. (2005) On a Fuzzy Kähler-Like Manifold Which Is Consistent with the Two Slit Experiment. International Journal of Nonlinear Sciences and Numerical Simulation, 6, 95-98. http://dx.doi.org/10.1515/ijnsns.2005.6.2.95

[46] Finkelstein, D.R. (1996) Quantum Relativity. A Synthesis of the Ideas of Einstein and Heisenberg. Springer, Berlin, Germany.

[47] El Naschie, M.S. (2006) Elementary Prerequisites for E-Infinity (Recommended Background Readings in Nonlinear Dynamics, Geometry and Topology). Chaos, Solitons \& Fractals, 30, 579-605. http://dx.doi.org/10.1016/j.chaos.2006.03.030

[48] El Naschie, M.S. (2004) The Concepts of E-Infinity: An Elntary Intrction to the Cantorian-Fractal Theory of Quantum Physics. Chaos, Solitons \& Fractals, 22, 495-511. http://dx.doi.org/10.1016/j.chaos.2004.02.028

[49] El Naschie, M.S. (2007) A Review of Application and Results of E-Infinity. International Journal of Nonlinear Science \& Numerical Simulation, 8, 11-20. http://dx.doi.org/10.1515/IJNSNS.2007.8.1.11

[50] El Naschie, M.S. (2002) On a Class of General Theories for Higher Energy Particle Physics. Chaos, Solitons \& Fractals, 14, 649-668. http://dx.doi.org/10.1016/S0960-0779(02)00033-4

[51] El Naschie, M.S. (1998) Superstrings, Knots and Noncommutative Geometry in E-Infinity Space. International Journal of Theoretical Physics, 37, 2935-2951. http://dx.doi.org/10.1023/A:1026679628582

[52] El Naschie, M.S. (1991) Quantum Mechanics and the Possibility of a Cantorian Spacetime. Chaos, Solitons \& Fractals, 1, 485-487. http://dx.doi.org/10.1016/0960-0779(91)90019-6

[53] El Naschie, M.S. (1994) Is Quantum Space a Random Cantor Set with a Golden Mean Dimension at the Core? Chaos, Solitons \& Fractals, 4, 177-179. http://dx.doi.org/10.1016/0960-0779(94)90141-4

[54] El Naschie, M.S. (2005) A Guide to the Mathematics of E-Infinity Cantorian Spacetime Theory. Chaos, Solitons \& Fractals, 25, 955-964. http://dx.doi.org/10.1016/j.chaos.2004.12.033

[55] El Naschie, M.S. (1996) Time Symmetry Breaking, Duality and Cantorian Spacetime. Chaos, Solitons \& Fractals, 7, 499-518. http://dx.doi.org/10.1016/0960-0779(96)00007-0

[56] El Naschie, M.S. (2004) Quantum Gravity, Clifford Algebra, Fuzzy Set Theory and the Fundamental Constants of Nature. Chaos, Solitons \& Fractals, 20, 297-330. http://dx.doi.org/10.1016/j.chaos.2003.09.029

[57] El Naschie, M.S. (2009) The Theory of Cantorian Spacetime and High Energy Particle Physics (An Informal Review). Chaos, Solitons \& Fractals, 41, 2635-2646. http://dx.doi.org/10.1016/j.chaos.2008.09.059

[58] El Naschie, M.S. (2005) From Experimental Quantum Optics to Quantum Gravity via a Fuzzy Kähler Manifold. Chaos, Solitons \& Fractals, 25, 969-977. http://dx.doi.org/10.1016/j.chaos.2005.02.028

[59] El Naschie, M.S. (2006) Topics in the Mathematical Physics of E-Infinity Theory. Chaos, Solitons \& Fractals, 30, 
656-663. http://dx.doi.org/10.1016/j.chaos.2006.04.043

[60] El Naschie, M.S. (2000) On the Unification of Heterotic Strings, M Theory and E ( $\infty$ ) Theory. Chaos, Solitons \& Fractals, 11, 2397-2408. http://dx.doi.org/10.1016/S0960-0779(00)00108-9

[61] El Naschie, M.S. (2006) Elementary Number Theory in Superstring Loop Quantum Mechanics, Twistors and E-Infinity High Energy Physics. Chaos, Solitons \& Fractals, 27, 297-330. http://dx.doi.org/10.1016/j.chaos.2005.04.116

[62] El Naschie, M.S. (2009) Wild Topology, Hyperbolic Geometry and Fusion Algebra of High Energy Particle Physics. Chaos, Solitons \& Fractals, 13, 1935-1945. http://dx.doi.org/10.1016/S0960-0779(01)00242-9

[63] El Naschie, M.S. (2004) Quantum Gravity from Descriptive Set Theory. Chaos, Solitons \& Fractals, 19, $1339-1344$. http://dx.doi.org/10.1016/j.chaos.2003.08.009

[64] El Naschie, M.S. (1995) A Note on Quantum Mechanics, Diffusional Interference and Information. Chaos, Solitons \& Fractals, 5, 881-884. http://dx.doi.org/10.1016/0960-0779(95)00040-B

[65] El Naschie, M.S. (1993) On Dimensions Of Cantor Set Related Systems. Chaos, Solitons \& Fractals, 3, 675-685. http://dx.doi.org/10.1016/0960-0779(93)90053-4

[66] El Naschie, M.S. (1997) Fractal Gravity and Symmetry Breaking in a Hierarchical Cantorian Space. Chaos, Solitons \& Fractals, 8, 1865-1872. http://dx.doi.org/10.1016/S0960-0779(97)00039-8

[67] El Naschie, M.S. (1997) Remarks on Super Strings, Fractal Gravity, Nagasawa’s Diffusion and Cantorian Spacetime. Chaos, Solitons \& Fractals, 8, 1873-1886. http://dx.doi.org/10.1016/S0960-0779(97)00124-0

[68] El Naschie, M.S. (2003) Modular Groups in Cantorian E-Infinity High Energy Physics. Chaos, Solitons \& Fractals, 16, 353-366. http://dx.doi.org/10.1016/S0960-0779(02)00440-X

[69] El Naschie, M.S. (1995) Banach-Tarski Theorem and Cantorian Micro Spacetime. Chaos, Solitons \& Fractals, 5, 1503-1508. http://dx.doi.org/10.1016/0960-0779(95)00052-6

[70] El Naschie, M.S. (2006) Hilbert Space, the Number of Higgs Particles and the Quantum Two-Slip Experiment. Chaos, Solitons \& Fractals, 28, 9-13. http://dx.doi.org/10.1016/j.chaos.2005.05.010

[71] El Naschie, M.S. (2006) The Idealized Quantum Two-Slit Gedanken Experiment Revisited-Criticism and Reinterpretation. Chaos, Solitons \& Fractals, 27, 843-849. http://dx.doi.org/10.1016/j.chaos.2005.06.002

[72] El Naschie, M.S. (2003) The VAK of Vacuum Fluctuation, Spontaneous Self Organization and Complexity Theory Interpretation of High Energy Particle Physics and the Mass Spectrum. Chaos, Solitons \& Fractals, 18, 579-605.

[73] El Naschie, M.S. (2005) Non-Euclidean Spacetime Structure and the Two-Slit Experiment. Chaos, Solitons \& Fractals, 26, 1-6. http://dx.doi.org/10.1016/j.chaos.2005.02.031

[74] El Naschie, M.S. (2006) Hilbert, Fock and Cantorian Spaces in the Quantum Two-Slit Gedanken Experiment. Chaos, Solitons \& Fractals, 27, 39-42. http://dx.doi.org/10.1016/j.chaos.2005.04.094

[75] El Naschie, M.S. (2006) On an Eleven Dimensional E-Infinity Fractal Spacetime Theory. International Journal of Nonlinear Sciences \& Numerical Simulation, 7, 407-409.

[76] El Naschie, M.S. (2006) Fuzzy Dodecahedron Topology and E-Infinity Spacetime as a Model for Quantum Physics. Chaos, Solitons \& Fractals, 30, 1025-1033. http://dx.doi.org/10.1016/j.chaos.2006.05.088

[77] El Naschie, M.S. (2006) On Two New Fuzzy Kähler Manifolds, Klein Modular Space and ’t Hooft Holographic Principles. Chaos, Solitons \& Fractals, 29, 876-881. http://dx.doi.org/10.1016/j.chaos.2005.12.027

[78] El Naschie, M.S. (2003) Complex Vacuum Fluctuation as a Chaotic "Limit” Set of Any Kleinian Group Transformation and the Mass Spectrum of High Energy Particle Physics via Spontaneous Self Organization. Chaos, Solitons \& Fractals, 17, 631-638. http://dx.doi.org/10.1016/S0960-0779(02)00630-6

[79] El Naschie, M.S. (2006) Superstrings, Entropy and the Elementary Particles Content of the Standard Model. Chaos, Solitons \& Fractals, 29, 48-54. http://dx.doi.org/10.1016/j.chaos.2005.11.032

[80] El Naschie, M.S. (1999) Nuclear Spacetime Theories, Superstrings, Monster Group and Applications, Chaos, Solitons \& Fractals, 10, 567-580. http://dx.doi.org/10.1016/S0960-0779(98)00313-0

[81] El Naschie, M.S. (2011) Quantum Entanglement as a Consequence of a Cantorian Micro Spacetime Geometry. Journal of Quantum Information Science, 1, 50-53. http://dx.doi.org/10.4236/jqis.2011.12007

[82] El Naschie, M.S. (2004) The Symplictic Vacuum, Exotic Quasi Particles and Gravitational Instanton. Chaos, Solitons \& Fractals, 22, 1-11. http://dx.doi.org/10.1016/j.chaos.2004.01.015

[83] El Naschie, M.S. (1998) COBE Satellite Measurement, Hyper Spheres, Superstrings and the Dimension of Spacetime. Chaos, Solitons \& Fractals, 9, 1445-1471. http://dx.doi.org/10.1016/S0960-0779(98)00120-9

[84] El Naschie, M.S. (2006) Advanced Prerequisites for E-Infinity Theory. Chaos, Solitons \& Fractals, 3, 636-641. 
http://dx.doi.org/10.1016/j.chaos.2006.04.044

[85] El Naschie, M.S. (2015) The Casimir Topological Effect and a Proposal for a Casimir-Dark Energy Nano Reactor. World Journal of Nano Science \& Engineering, 5, 26-33. http://dx.doi.org/10.4236/wjnse.2015.51004

[86] El Naschie, M.S. (2015) Kerr Black Hole Geometry Leading to Dark Matter and Dark Energy via E-Infinity Theory and the Possibility of Nano Spacetime Singularity Reactor. Natural Science, 7, 210-225. http://dx.doi.org/10.4236/ns.2015.74024

[87] El Naschie, M.S. (2014) Why E Is Not Equal $m c^{2}$. Journal of Modern Physics, 5, 743-750. http://dx.doi.org/10.4236/jmp.2014.59084

[88] El Naschie, M.S. (2014) On a New Elementary Particle from the Disintegration of the Symplectic 't Hooft-VeltmanWilson Fractal Spacetime. World Journal of Nuclear Science and Technology, 4, 216-221. http://dx.doi.org/10.4236/wjnst.2014.44027

[89] El Naschie, M.S. (2014) From $E=m c^{2}$ to $E=m c^{2} / 22$-A Short Account of the Most Famous Equation in Physics and Its Hidden Quantum Entangled Origin. Journal of Quantum Information Science, 4, 284-291.

http://dx.doi.org/10.4236/jqis.2014.44023 


\section{Appendix 1}

The present analysis and derivation depends fundamentally upon the von Neumann-Connes recursive dimensional function [25] [32]

$$
D=a+b \phi, \quad a, b \in Z \text { and } \phi=2 /(\sqrt{5}+1)
$$

This function was used to describe superficially pathological $x$ spaces such as that of Penrose tiling in noncommutative geometry [33] [34]. Subsequently the present author demonstrated that the Penrose fractal tiling may be viewed as a compactified Klein modular curve with $D=336+16 k \simeq 339$ degrees of freedom and that as such it is generic and represents the surface or the holographic boundary of a universe described in bulk by E8E8 exceptional Lie groups of super strings [57]. The dimensionality conservation equation connecting E8E8 with the Klein-Penrose boundary may be given in various forms of which the following is the simplest [19] [43] [49]

$$
|E 8 E 8|=|S L(2,7)|+|S U(2)|+\bar{\alpha}_{o}+G=336+3+137+20=496
$$

where $\bar{\alpha}_{o}=137$ is the inverse electromagnetic fine structure constant and $G=20$ is the degrees of freedom of pure gravity in $D=8$ super space or alternatively, the number of the independent components of the Riemannian curvature tensor in $D=4$ Einstein space [43] [49].

\section{Appendix 2}

The following illustrates and derives the basic results of cobordism as applied to our theory in an elementary fashion. We start from a three dimensional cube. The surfaces of the cube are evidently square, i.e. two dimensional. This means we have an equation stating that [3]-[16]

$$
D=n-1
$$

where $n=3$ for a cube and it follows then that its "borders" or surface have $D=3-1=2$. It is trivial to see that the same will go on for the square where the "borders" are one dimensional lines

$$
D=2-1=1 \text {. }
$$

For a line the "borders" are the end points so that our elementary equation still holds

$$
D=1-1=0 .
$$

The next step is on the other hand not trivial. We ask our self what is the border" or the surface of a point? The point is a zero dimensional object, which in theory is the best model of a pre-quantum particle and now we are de facto asking what is the dimension of the neighbourhood of a zero point? Our equation says then that it is

$$
D=0-1=-1 \text {. }
$$

This is exactly how K. Menger and P. Urysohn defined the empty set [36] [37]. The present author, following various ideas partially connected to Mandelbrot's notion of the degrees of emptiness of an empty set, then reasoned that the total insubstantial nothingness is neither the zero set $D(0) \equiv(0, \phi)$ nor the empty set $D(-1) \equiv\left(-1, \phi^{2}\right)$ but the completely empty set $D(-\infty) \equiv(-\infty, 0)$ [20] [26] [39] [40]. 